\title{
Interference Detection Algorithm of Aircraft Components Assembly based on Measured Data
}

\author{
Wei Zhang ${ }^{1, a}$, Luling $A n^{1, b}$, Qilin Jiang ${ }^{1, c}$, Ruiheng $\mathrm{Xiao}^{2, \mathrm{~d}}$ \\ ${ }^{1}$ College of Mechanical and Electrical Engineering, Nanjing University of Aeronautics and \\ Astronautics, Nanjing, 210016, China \\ ${ }^{2}$ Institute of Aeronautical Technology, Shanghai Aircraft Manufacturing, Shanghai, 200436, China \\ azwsmile12@163.com, banllme@nuaa.edu.cn, '635562388@qq.com, ${ }^{\mathrm{d}} 237203174 @ q q . c o m$
}

Keywords: Point cloud; Assembly; Feature; Interference detection; Algorithm

\begin{abstract}
Parts manufacture deviation may cause interference in aircraft components assembly, and the handling of interference is a complex work. In order to solve this problem, the paper proposes an interference detection method based on measured data. First, the assembly feature was obtained by fitting the point cloud (PC) data, then the feature was used to align PC model with theoretical assembly model, so that the PC model could be placed at theoretical position. Furthermore, individual parts were marked and paired in accordance with whether they form interfaces (only direct surface-to-surface contact between components), and the interfaces were regionally partitioned from the complete model. Finally, a series of parallel sections were used to perform intersection operation on the fitted surfaces, and the intersecting point set on each section needed curve fitting so as to calculate the interference and clearance values. This method can quickly calculate the interference distribution of actual assembly parts. Based on the interference, the handling of follow-up assembly could be guided quantitatively. The feasibility and validity of this method are demonstrated by an aircraft wing box case, proving that it can improve the efficiency of aircraft components assembly.
\end{abstract}

\section{Introduction}

Aircraft products are of complex structures, large spatial sizes, low stiffness and numerous parts. When it comes to assembly, the interference caused by parts manufacture deviation and the installation error of fixtures, as well as the position error of parts are frequently generated. Among the above problems, the manufacture deviation of parts is inevitable. Even if the assembly tolerance is maintained for theoretical models, the parts are still possible to impose assembly interference owing to their manufacture deviation. In this occasion, certain parts are in need of fettling or position adjustment so that the assembly can be conducted smoothly. Nonetheless, repeated trial assembly and fettling require a drastic amount of time and workforce, tremendously affecting the efficiency and costs in aircraft assembly.

Modern aircrafts tend to have higher standards for lightweight, economy and safety, which increasingly popularize the application of composites in the aircraftmanufacturing industry [1]. The introduction of composites for the manufacturing of aircraft structures allows reducing weight, enhancing structural stiffness and cutting down fuel consumption. As for large-scale composite structures, however, owing to the characteristics of their manufacture process, along with the complexity of their structures and shapes, considerable deviations can be found in their geometric appearances as well as dimensions. Yet, the high performance of aircraft requires assembly tolerances to be tightened. Once interference or clearance occurs, the fixation of parts on the theoretical positions by forceful assemblies shall lead to a series of problems, such as extra assembly stress on the structure and the decrease in the sealing effect, etc. This opens the need for positioning measured data and discovering interference before physical assembly.

Hence, this paper proposed an interference detection method for assembly, which need to acquire the 3D PC data of parts through measurement. In this approach, the posture of a PC model is identified by assembly features. Accordingly, the value and distribution of interference between 
assembly parts are calculated. Based on this, the handling of follow-up assembly is guided quantitatively.

In terms of interference detection, space partitioning [2] divides the detection space into a series of cells, and only the geometric objects in the same cell are performed intersection operation. This method needs enormous storage space and has low algorithmefficiency. Hierarchical bounding box [3] is constructed to approach geometry solid. The components which are impossible to cause interference are removed, but the constructing process requires complex subdivision or merge algorithm, and has low efficiency. Another template based interference detection algorithm [4] takes template as reconstruction model to take part in the interference analysis, but the manufacture deviation is not taken into account. So the main theme of this paper is putting forward a fast calculation method of interference based on measured data, and upon the distribution of interference, engineers could handle the parts before physical assembly.

\section{Assembly features fitting and model location}

In this paper, an aircraft wing box is taken as an example. First, the PC data of parts (panel, spar, rib) need to be obtained by scanning, then assembly features are applied to find the position of PC model, after that the calculation of interference can be carried out. As for the wing box, there is no direct assembly-constrained relationship between individual components, which are merely positioned by assembly jigs. The PC data reflects the geometric information, but there is still the necessity of identifying their postures before they can be used in the analysis of interference. Thereby, based on the measured PC, models with assembly features are constructed. The processes of interference detection are shown in Fig. 1.

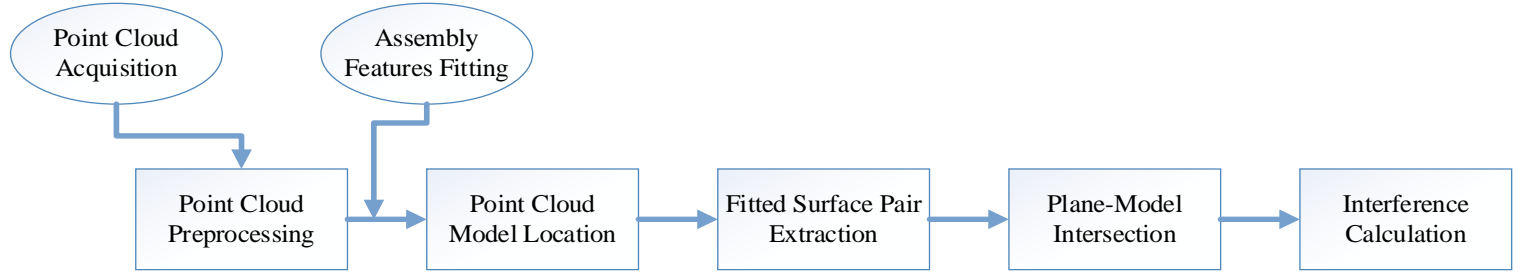

Fig. 1. The processes of interference detection.

An indirect location approach is applied on PC model, that is, we use assembly features to align PC model with theoretical assembly model. The term assembly feature is indicative of an associative set of geometric factors that possess certain engineering significance as well as the capability of generating assembly-constrained relationships (such as aligned, fitted, coaxial and tangent, etc.) [5]. It provides services for the assembly relationship with certain geometric structure as a carrier. The geometric characteristics that form the assembly features mainly involve point, line and plane. The PC models require region-segmentation to fit assembly features. This paper constructs features through the application of local reverse engineering, and in contrast, non-assembly features are not fitted. For the wing box, assembly features includ the plane and the cylinder of hole, so a face-based partition method is appropriate. The points having the same or similar partial differential properties belong to a same surface, then use least square method to fit features. Fig. 2 demonstrates the assembly features on the PC model of wing box front spar, which are the plane and the axes of holes.

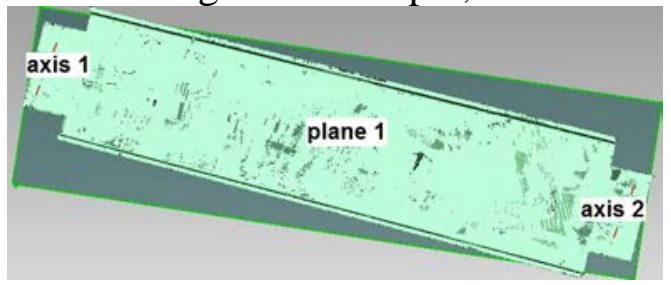

Fig. 2. The fitted assembly features of the wing box front spar.

The fitting process of assembly features may cause errors. There are two main factors which may generate the reverse error, one is measuring error, and another is constructing error. The former is caused by scanning machine, as for the Faro Edge 2.7 used in this case, the space maximum deviation is $0.034 \mathrm{~mm}$. The latter is mainly resulted from PC fitting, and take the plane fitting as an example, the 
overall error range is about $\pm 0.026 \mathrm{~mm}$, based on multiple attempts on trial. By using root mean square error synthesis, the final total error can be calculated as follow:

$$
\Delta_{\text {total }}=\sqrt{\Delta_{\text {measuring }}^{2}+\Delta_{\text {reversing }}^{2}}=\sqrt{\left(0.034^{2}+0.026^{2}\right)} \approx 0.042 \mathrm{~mm} .
$$

\section{Interference calculation}

After identifying the postures of PC models in the assembly space using assembly features, the interference value of parts in the theoretical assembly position can be calculated with the PC-based static algorithm of interference detection. Both assembly parts can only generate interference on the surfaces that form an interface, which means other surfaces do not affect the interference results. Hence, in order to simplify the calculation, only the interfaces of two parts that form direct surface-to-surface contact are extracted and given the name of fitted surface pair as well as have their interference value calculated directly.

\subsection{Fitted surface pair extraction}

First of all, individual parts are marked and paired in accordance with whether they form interface with components. Those parts that form interfaces are marked as Part $_{1}-A$, Part $_{1}-B ; \ldots ;$ Part $_{n}-A$, $\operatorname{Part}_{n}-B$. A pair of fitting components are regionally partitioned with an edge-based partition method [6], according to the curvature variation of data points. The points that have larger variations of curvature value served as boundary points that divide the whole PC model into several independent point set regions. The surfaces formed interfaces are extracted by man-machine interaction, with other surfaces that are impossible to generate interference excluded. Such fitted surface pairs are then stored as new Part 1 - $a$, Part 1 -b models. The upper panel and front spar are shown as a pair of fitting components in Fig. 3 (left). It is observed from the assembly process that in the theoretical assembly position, the inner surface of the upper panel and the upper flange of the front spar are required to be fitted. However, the fitted surfaces may introduce interference or clearance. Therefore, the inner surface and the upper flange are partitioned from the complete model and stored as new models, see Fig. 3 (right).
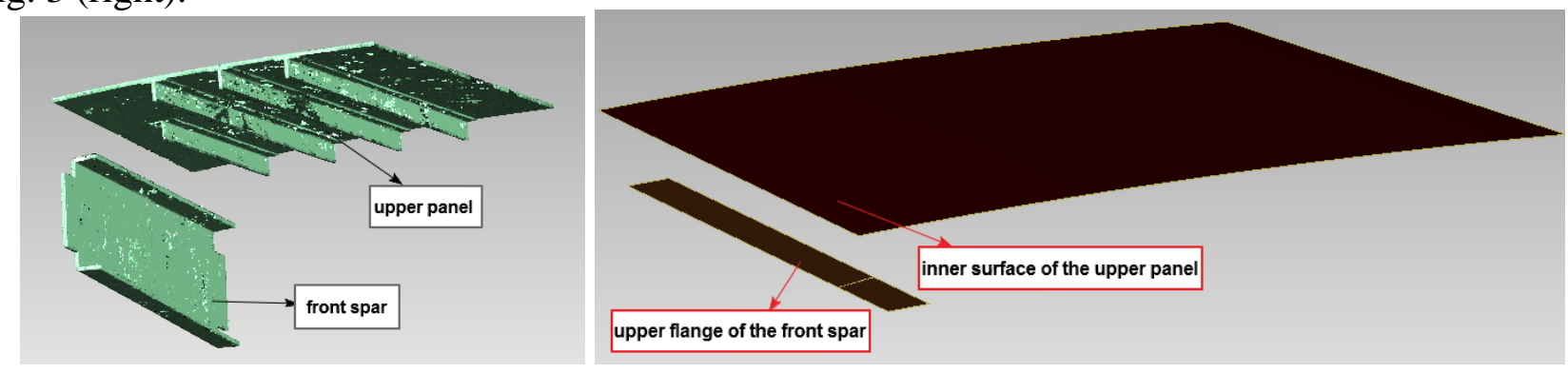

Fig. 3. A pair of fitted components (left) and the fitted surfaces (right)

\subsection{Plane-model intersection}

Providing that the relative positions of two components remains unchanged, the distributions of interference and clearance between components will not be affected. Thereby, with the intention to simplify the calculation, a local coordinate system named $\mathrm{O}-\mathrm{XYZ}$ is built. Take the assembly of the front spar and upper panel as an example, the parallel plane of the spar web plate is XOY, with axis Z perpendicular to the web plate. The local coordinate system is illustrated in Fig. 4.

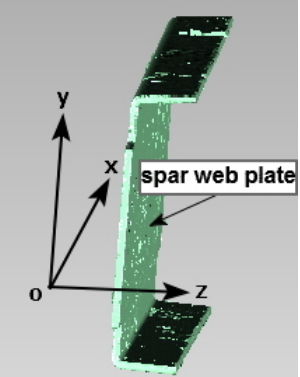

Fig. 4. The local coordinate system O-XYZ. 
The choice of direction for the calculation of sections is associated to the quality of the section curve established at the end. Hence, the priority is to determine the calculation direction according to the curvature variation of the fitted surface pairs. If the surface is relatively flat and with unapparent curvature variations, calculation direction will not impose remarkable impact on the section curve. Nonetheless, if the curvature variation of one direction is more significant than the others, the calculation direction ought to be perpendicular to it. In the example of this paper, a larger curvature variation of the fitted surface pair is found in the axis Z. Hence, axis X, which is perpendicular to it, is chosen as the calculation direction. Then, use the calculation direction as a vector of section plane method to perform intersection operation on the fitted surface pair so as to calculate the section curves on a random plane $E$.

As PC data are discrete finite sets of points, it is hardly possible to strictly determine their section curves on plane $E$. Therefore, it is necessary to set a section step size $\delta$. Following the direction of the normal vector on plane $E$, parallel planes $E_{l}$ and $E_{r}$ are generated from an equidistance of $\delta / 2$, as shown in Fig. 5. Among the data, points located between $E_{l}$ and $E_{r}$ are chosen as usable points to generate the section curve of the part.

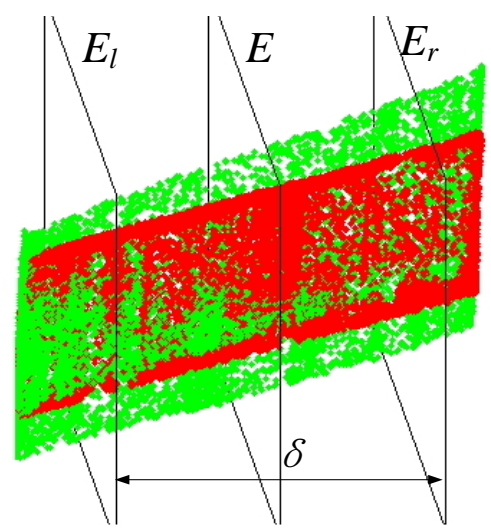

Fig. 5. Schematic diagram of section planes.

The value of the section step size $\delta$ is related to the quality of section curve that is worked out and can be set according to the density $\rho$ of PC model. The density $\rho$ can be calculated by $n$ sample points $(S)$ randomly taken out from the whole data set, and $S=\left\{P_{i}, i=1, \ldots, n\right\}$. Then find $m$ points having the nearest distance from $P_{i}$, and mark the distance as $D_{i}=\left\{d_{i}^{j}, j=1, \ldots, k\right\}$. Thereby, the density $\rho$ of PC can be given by

$$
\rho=\frac{\sum_{i=1}^{n} \sum_{j=1}^{k} d_{i}^{j}}{n \times k}
$$

The section step size $\delta$ can be derived from the density $\rho$, that is $\delta=k \rho$. Multiple trials prove that the section curve may have good quality when $k=4 \sim 8$.

\subsection{Data separation and intersection seeking}

Based on the section step size $\delta$, points between plane $E_{l}$ and $E_{r}$ are found out, which also are divided into two parts: $K_{l}$ and $K_{r}$. That is, calculate the signed distance $d_{i}$ from $P_{i}$ in the whole data set to section $E$, and $d_{i}=A x_{i}+B y_{i}+C z_{i}+D$. If $0 \leq d_{i} \leq \delta / 2$, then $P_{i} \in K_{l}$; other if $-\delta / 2 \leq d_{i}<0$, then $P_{i}$ $\in K_{r}$. Both $K_{l}$ and $K_{r}$ are two files for PC data storage. The following step is seeking the intersection on section plane $E$, see Fig. 6 . For any point $P_{l i}$ in $K_{l}$ find $P_{r i}$ in $K_{r}$ which has the nearest distance from $P_{l i}$, and make line $l$ pass the two points $P_{l i}$ and $P_{r i}$. 


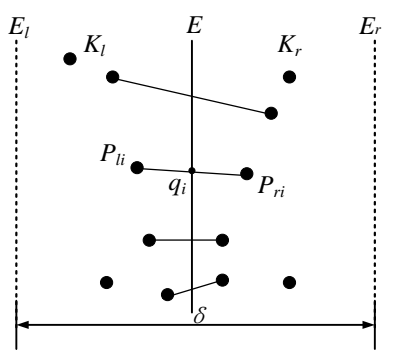

Fig. 6. Intersection seeking between section plane and point cloud

After that, the intersection of line $l$ and section plane $E$ is arrived at $q_{i}\left(x_{i}, y_{i}, z_{i}\right)$. The equations of line $l$ and $x_{i}$ are given by

$$
\begin{aligned}
& \frac{x_{i}-x_{l i}}{x_{r i}-x_{l i}}=\frac{y_{i}-y_{l i}}{y_{r i}-y_{l i}}=\frac{z_{i}-z_{l i}}{z_{r i}-z_{l i}} \\
& x_{i}=x_{\text {min }}+(m+0.5) \delta
\end{aligned}
$$

The $x_{\min }$ is the minimum in axis $\mathrm{X}$ among the whole data set, and $m$ is the number of section planes, $m=(0, \ldots, \theta-1)$. Finally, intersection $q_{i}$ is saved into the file listE as output data. If point $P_{r i}$ that corresponds to point $P_{l i}$ cannot be found in file $K_{r}, P_{l i}$ will be invalid. As soon as the calculation of one section is completed, move one step size $\delta$ forward and repeat the above calculation until the whole model has been calculated. By calculating model 2 after finishing the calculation of model 1, the distribution of PC data on each section plane can be effortlessly derived. The calculation procedures are shown in Fig. 7.

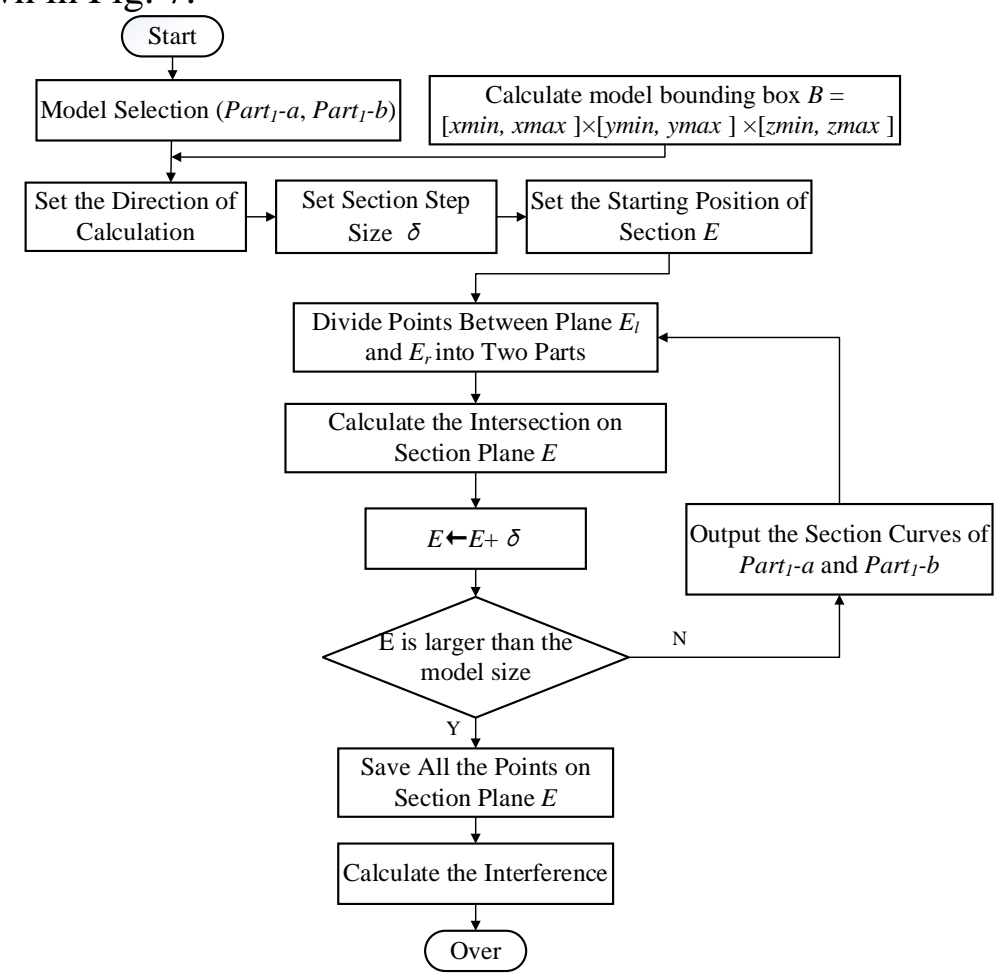

Fig. 7. Interference calculation procedures.

\subsection{Interference value calculation}

The PC data obtained by scanning and measuring the actual components are mass discrete point clouds [7]. Therefore, the intersections of the section planes and the PC data are scattered points. Such points reflect the geometric properties of the parts, but the interference value of the fitted surfaces between two components is not apparent. The points are in need of curve matching in order to derive the outline of both components on the section plane, which is then used in the calculation of interference and clearance values. Least square method is the most commonly employed means in curve matching. It derives a linear function minimising the quadratic sum of the error between the scattered data points and the matched curve, through solving which the matched curve can be worked 
out. The interference value of two assembly parts at a certain location is illustrated in Fig. 8. Point A on line 1 and the normal at $\mathrm{A}$ intersects line 2 at $\mathrm{B}$. The length of $\mathrm{AB}$ is the interference at point $\mathrm{A}$. Mark positive value as interference and negative value as clearance.

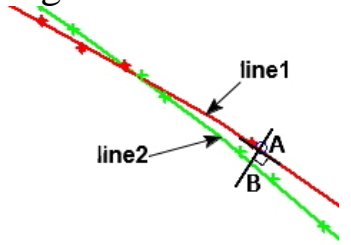

Fig. 8. Schematic diagram of interference value

\section{Case study: interference detection based on measured data}

Based on the algorithm proposed in this paper, corresponding calculation programmes are developed using MATLAB. Such programme is capable of calculating the interference value in any location of section planes of both assembly parts. It also provides details of the distribution of interference between components. Take the fitting of the upper panel and front spar in the wing box assembly as an example. Firstly, we scan and measure the actual component to obtain PC data, and pre-process the measured data, including denoising, simplifying and multi-view merging, etc., to generate a high quality PC model. Secondly, we use matched assembly features to align PC model with theoretical assembly model. The assembly positioning results of the front spar and the upper panel are illustrated in Fig. 9 (left). Finally, partition the fitted surfaces of both assembly parts and save them as Part $_{1}-a$, Part $_{1}$-b, see Fig. 9 (right).
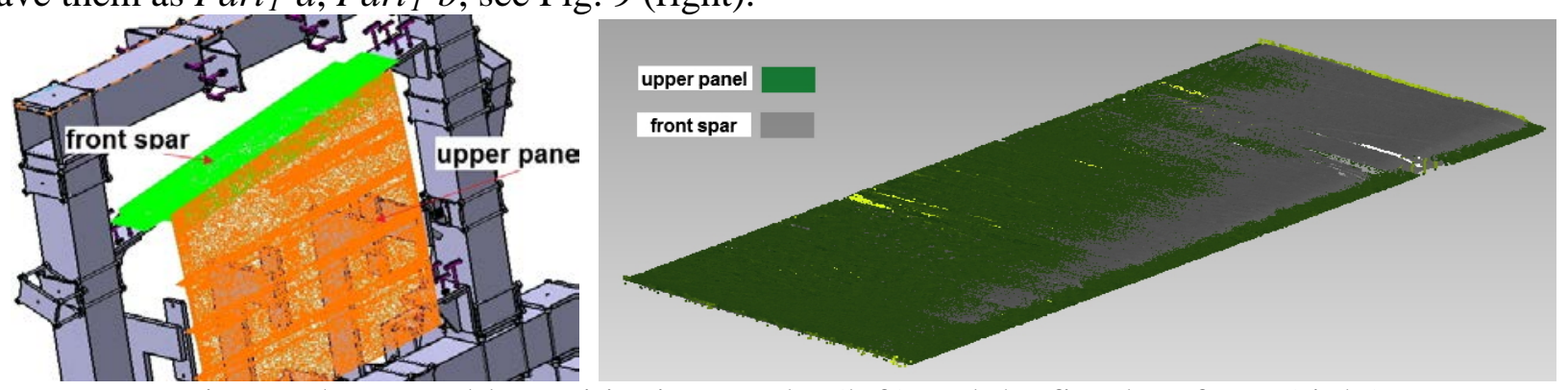

Fig. 9. The assembly positioning results (left) and the fitted surfaces (right)

The operational interface of the prototype system of the interference detection algorithm is shown in Fig.10 (left). The major functions of such operational interface involve the input and output of PC data, the intersection between PC and section plane, along with the analysis of the interference on sections. Fig.10 (right) displays the interference values of both components at three randomly appointed positions on the section plane.
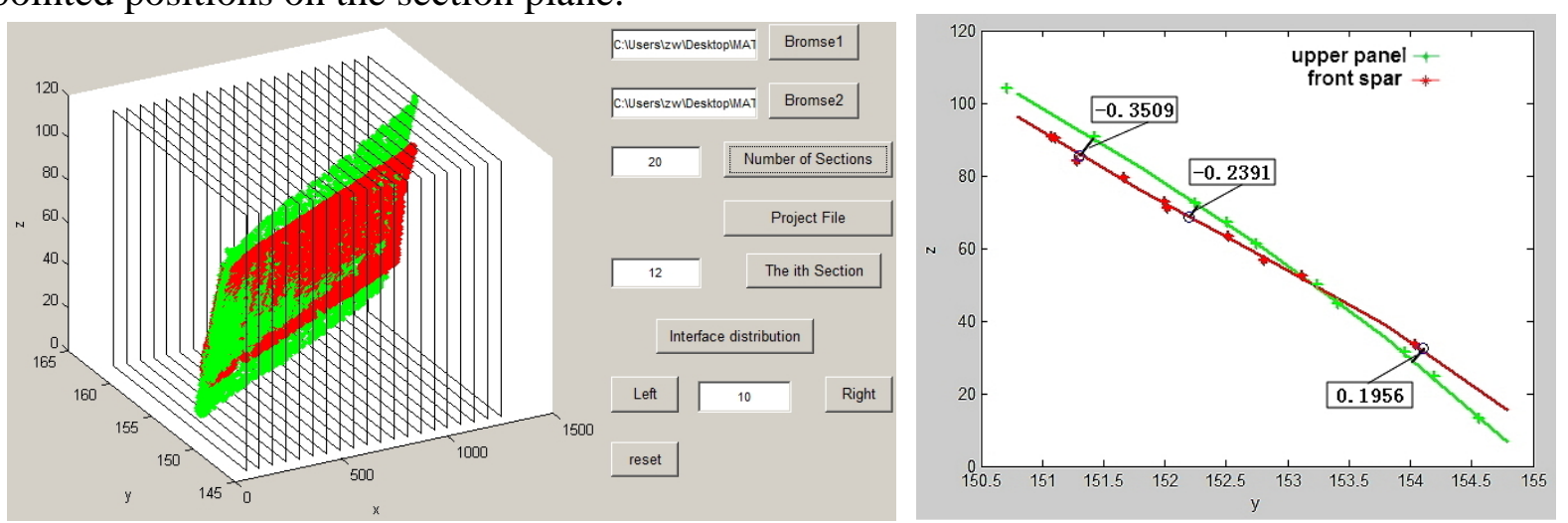

Fig. 10. The operational interface (left) and the interference values (right)

\section{Conclusions}

In this work an approach for interference detection of aircraft components assembly is presented and carried out based on measured data. Furthermore, we use the assembly features constructed 
through the application of local reverse engineering to identify the postures of PC, so that the actual surface information of the parts can be preserved to a maximum extent. Finally, the fitted surfaces of both assembly parts are cut by a series of parallel sections, so that the section curves on every cutting-plane can be calculated out. Based on the section curves, the interference distribution of assembly parts is apparently represented. This method not only simplify the interference calculation, but also give quantitatively the interference and clearance of assembly parts.

The implementation of the presented method on aircraft assembly offers a high potential for reducing the cycle times and improving the assembly quality.

\section{Acknowledgements}

This work is supported by Innovation Foundation of National Commercial Aircraft Manufacturing Engineering Technology Center of China (SAMC13-JS-15-021) and National Defense Basic Scientific Research Project (A0520132008).

\section{References}

[1] Burka P. Automated Gap Analysis and Adhesive Application during Fuselage Assembly. Conference Proceedings of the 6th International CFK-Valley Stade Convention 2012.

[2] JIMNEZ J P, THOMAS F, TORRAS C. 3D collision detection: A survey [J]. Computers and Graphics, 2001, 25(2): 269-285.

[3] Jiang Hong, Hou Jianbo, et al. Optimizing algorithm of interference checking and its implement in vehicle electric-brake virtual assembly system [J]. Chinese Journal of Mechanical Engineering, 2007, 43(12): 59-63. (in Chinese)

[4] Zhang $\mathrm{Xu}$, et al. Template Based Interference Detection Technology of Large Components Assembly [J]. Journal of Mechanical Engineering, 2010, 01: 147-153+161. (in Chinese)

[5] Zhang Hongxing, Xu Min, Jiang Zuhua. Study on the Definition and Representation of Assembly Features for Automatic Assembly [J]. Mechanical Science and Technology for Aerospace Engineering, 2005, 07: 824-826+851. (in Chinese)

[6] Ou Xinliang, Kuang Xiaolan, Ni Wenyin. Summarization on 3D Scattered Point Cloud Segmentation [J]. Journal of Hunan University of Technology, 2010, 05: 45-49. (in Chinese)

[7] Seokbae Son, et al. Automated Laser Scanning System for Reverse Engineering and Inspection. International Journal of Machine Tools \& Manufacture, 2002, 42: 889-897. 